\title{
Caracterización físico-mecánica de la madera de Eucalyptus camaldulensis para uso estructural proveniente de Restinga, Brasil
}

\author{
Physical-mechanical characterization of Eucalyptus camaldulensis wood for \\ structural utilization from Restinga, Brazil
}

Marta C. J. A. Nogueira', Victor A. De Araujo², Juliano S. Vasconcelos ${ }^{3}$, Carlos M. Gutiérrez-Aguilar ${ }^{4}$, José N. da Cruz ${ }^{5}$, Julio C. S. Vasconcelos ${ }^{6}$, Fábio Prataviera ${ }^{6}$, André L. Christoforo y Francisco A. R. Lahr ${ }^{8, *}$

\begin{abstract}
Resumen
La biomasa para energía y los productos a base de madera sólida son algunas de las aplicaciones más populares del Eucalyptus camaldulensis Dehnh. Sin embargo, esta especie todavía es subutilizada en la construcción civil por toda América Latina. Los objetivos de este estudio fueron la evaluación de las propiedades del Eucalyptus camaldulensis para determinar y ampliar su utilización para elementos estructurales. Las maderas fueron procedentes de plantaciones en la ciudad de Restinga, Estado de São Paulo, Brasil. Dos propiedades mecánicas y catorce físicas fueron evaluadas para la caracterización de la madera de Eucalyptus camaldulensis con soporte de la norma brasileña NBR 7190 (1997). Dos contenidos de humedad fueron observados, el primero en el punto de saturación de las fibras a 30\% y el segundo en el punto estándar a $12 \%$ de acuerdo con la norma brasilera al contenido de humedad de equilibrio. Este estudio evaluó 538 repeticiones. Finalmente, los resultados obtenidos fueron analizados estadísticamente mediante la prueba t con $5 \%$ de significancia. Diez propiedades de resistencia indicaron una mejora en sus propiedades durante la reducción del contenido de humedad estudiado: compresión paralela y normal, tensión normal y flexión en los módulos de ruptura; compresión normal y flexión en los módulos de elasticidad; durezas normal y paralela; cizallamiento; y agrietamiento. Así, el Eucalyptus camaldulensis puede ser utilizado para fin estructural, debido a la mejora de sus propiedades mecánicas con la reducción de humedad y por presentar valores de propiedades mecánicas conformes a la norma brasileña.
\end{abstract}

Palabras clave: Eucalipto rojo, eucalipto, madera, resistencia mecánica, construcción.

\footnotetext{
${ }^{1}$ Departamento de Arquitectura, Universidad Federal de Mato Grosso (UFMT), Brasil. ${ }^{2}$ Departamento de Ciencias Forestales, Universidad de São Paulo (USP), Brasil. ${ }^{3}$ Facultad de Ciencias Agronómicas, Universidad Estadual Paulista (UNESP), Brasil. ${ }^{4}$ Departamento de Diseño, Instituto Tecnológico Metropolitano (ITM), Colombia. ${ }^{5}$ Departamento de Estadística, Universidad Federal do Mato Grosso (UFMT), Brasil. ${ }^{6}$ Departamento de Ciencias Exactas, Universidad de São Paulo (USP), Brasil. ${ }^{7}$ Departamento de Ingeniería Civil, Universidad Federal de São Carlos (UFSCar), Brasil. ${ }^{8}$ Departamento de Ingeniería de Estructuras, Universidad de São Paulo (USP), Brasil. *Autor de Correspondencia: frocco@sc.usp.br
} 


\begin{abstract}
Biomass for energy and products based on solid wood are some of the most popular applications of Eucalyptus camaldulensis Dehnh. However, this species is still underused in construction throughout Latin America. The objectives of this study were the evaluation of the properties of Eucalyptus camaldulensis to determine and expand its utilization for structural elements. Two physical and fourteen mechanical properties were evaluated for wood characterization of Eucalyptus camaldulensis with support of Brazilian standard NBR 7190 (1997). Woods were obtained from plantations from Restinga city, São Paulo state, Brazil. Two moisture contents were observed, the first in the fiber saturation point at $30 \%$, and the second in the standard point at $12 \%$ according to the Brazilian standard at equilibrium moisture content. This study evaluated 538 repetitions. Finally, the results obtained were statistically analyzed by the t-test at $5 \%$ of significance. Ten resistance properties indicated an improvement in their properties during the reduction of the studied moisture content: parallel and normal compression, normal traction and flexion in the rupture modules; normal compression and flexion in the modulus of elasticity; normal and parallel hardness; shearing; and cracking. Thus, Eucalyptus camaldulensis can be used for structural purposes, due to the improvement of its mechanical properties with the reduction of humidity and by presentation of mechanical property values conforming to Brazilian standard.
\end{abstract}

Key words: River Red Gum, eucalypt, timber, mechanical strength, construction.

\section{Introducción}

River Red Gum (Wallis 1970; Boland et al. 2006; IICA 2007) o eucalipto rojo (FAO/UN 2011) se refieren a los nombres comerciales, respectivamente en inglés y español, del $E$ ucalyptus camaldulensis Dehnh. var. camaldulensis, el cual se sitúa entre las diez especies más importantes de la industria australiana de la madera (FAO/UN 1981). Las principales áreas de aparición del Eucalyptus camaldulensis son entre las latitudes de $15,5^{\circ}$ a $38^{\circ} \mathrm{S}$, en altitudes variando de 30 a 600 metros, predominantemente en las márgenes de los ríos (Ferreira 1979). De copa grande y tronco grueso, el eucalipto rojo es un árbol de tamaño entre medio y alto, que supera los 20 metros de altura y diámetro entre 1 y 2 metros (Boland et al. 2006). Es una de las especies más adecuadas para zonas críticas de reforestación, en los cuales, problemas ligados al suelo y a deficiencias hídricas sean factores limitantes para otras especies (Ferreira 1979).

El Eucalyptus camaldulensis tiene la mayor variedad de cualquier plantación de eucalipto en Australia (FAO/UN 1981; Sun y Dickinson
1995; Dunlop et al. 2000; Catelotti et al. 2015), y es muy popular en Argelia, Chipre, Estados Unidos, Grecia, Irán, Italia, Jordania, Malta, México, Marrocos, Uruguay, (FAO/UN 1981), Argentina (Marlats y Pérez 1991; Lanfranco et al. 1999; Acuña et al. 2014; González et al. 2014), Chile (Sandoval et al. 2015), España (Conde et al. 1996; Verdeguer et al. 2009), India (Hunter 2001; Bedi et al. 2012), Tailandia (Pinyopusarerk et al. 1996; Ounban et al. 2016) y Zimbabue (Tyynelä 2001), sin embargo, también está presente en Angola, China, Colombia, Costa Rica, Ecuador, Etiopia, Gana, Israel, Costa de Marfil, Líbano, Lesoto, Mozambique, Perú, Túnez, Uganda (FAO/UN 1981), África del Sur (Dzikiti et al. 2016), Brasil (Sturion et al. 1987; Teixeira et al. 1995; Gonçalves y Passos 2000; Pagano y Scotti 2008), Nigeria (Onyewotu et al. 1994), Portugal (Durand et al. 2011; Pirralho et al. 2014), Tanzania (Chingaipe 1985; Sabas y Nshumbemuki 1988), Turquía (Aytar et al. 2011), etc.

El Eucalyptus camaldulensis es utilizado en la producción de aceites esenciales (Giamakis et al. 2001; Verdeguer et al. 2009; Knezevic et al. 
2016; Li et al. 2016), extractos solventes (Ashraf et al. 2015), ácidos y poli fenoles (Abd-Alla et al. 1980; Conde et al. 1996; Siddiqui et al. 2000; Medhi et al. 2010; Ikka et al. 2013), carbón activado (Patnukao et al. 2008; Patnukao y Pavasant 2008; Heidari et al. 2014), leña y carbón vegetal (Ferreira 1979; Sabas y Nshumbemuki 1988; Costa et al. 2017), pulpa de celulosa (Ohshima et al. 2005; Pirralho et al. 2014), barreras de vientos (Onyewotu et al. 1994), apicultura (FAO/UN 1981) y rehabilitación de áreas con alta concentración de salinidad (Kabay et al. 1986; Bell et al. 1993; Sun y Dickinson 1995; Bush et al. 2013). Dura y un poco densa, la madera del eucalipto rojo posee color rojizo y presenta veta entrecruzada, textura fina y bolsas de resina (Wallis 1970; FAO/UN 1981; Boland et al. 2006). Demuestra buena utilidad en la producción de durmientes, postes de cerca y eléctricos (Ferreira 1979), torneados, muebles, cercas, pisos, compensados, construcción, laminación (Boland et al. 2006), paneles de partículas de cemento-madera (Okino et al. 2004), viviendas y muelles (Wallis 1970), así como, para la obtención de aserrados (FAO/ UN 1981; González et al. 2014).

A pesar de su utilización en la construcción en muchos países desarrollados, el eucalipto rojo todavía posee baja utilización en América Latina, lo que amplía su potencial por ser una madera de buena calidad de fácil renovación forestal. La falta de literatura sobre sus propiedades puede contribuir a la limitación de su uso estructural más eficiente, en un momento que, según NAHB (1997), investigadores están buscando formas de hacer un uso más eficiente de las diferentes calidades y especies de madera. Frente a la exploración de nuevas posibilidades para reducir el uso de maderas nativas en la construcción, este estudio presenta la caracterización físico-mecánica del Eucalyptus camaldulensis procedente de plantación para su mayor uso estructural, con soporte de la norma brasileña de las estructuras, para dos diferentes contenidos de humedad.

\section{Materiales y Métodos}

Las muestras de eucalipto rojo fueron caracterizadas por la realización de 538 repeticiones para todos los ensayos físico-mecánicos, los cuales siguieron el mismo estándar considerado por Lahr et al. (2017) y Nogueira et al. (2018). Fueron utilizadas trozas de madera con diferentes diámetros y edades procedentes de plantaciones del Estado de São Paulo en Brasil (Cuadro 1).

Siendo así, catorce pruebas mecánicas y dos pruebas físicas fueron realizadas mediante las prescripciones de la norma brasileña NBR 7190 (1997) para las siguientes propiedades: densidad (básica y aparente), módulos de ruptura

\begin{tabular}{ccccc}
\hline Tronco $^{*}$ & Viga* & Edad & $\begin{array}{c}\text { Diámetro } \\
(\mathbf{m})\end{array}$ & Región** \\
\hline 1 & 2 & 41 & 0,260 & Restinga, Brasil \\
2 & 2 & 41 & 0,245 & Restinga, Brasil \\
3 & 2 & 41 & 0,230 & Restinga, Brasil \\
4 & 4 & 50 & 0,275 & Restinga, Brasil \\
5 & 2 & 50 & 0,270 & Restinga, Brasil \\
6 & 4 & 28 & 0,230 & Restinga, Brasil \\
7 & 2 & 28 & 0,200 & Restinga, Brasil \\
\hline
\end{tabular}

*cantidades; **ubicación de las trozas de madera recogidas para los ensayos

Cuadro 1. Detalles de las muestras de la madera de Eucalyptus camaldulensis procedentes de plantaciones. 
(tensiones paralela y normal, compresiones normal y paralela, flexión estática), módulos de elasticidad (compresiones normal y paralela, tensión paralela, flexión estática), dureza (paralela y perpendicular), cizallamiento, y el agrietamiento. Así, la madera fue evaluada con respecto a la reducción de humedad, de un punto de saturación de las fibras estable a 30\% (madera verde) para un punto estándar estable de $12 \%$ para estructuras de madera (madera seca), indicado por la norma ABNT NBR 7190 (1997), al contenido de humedad de equilibrio. Además, según García Meza et al. (2017), es importante relacionar el comportamiento físico y mecánico para proponer las aptitudes de uso. Por eso, este trabajo también listó posibles usos constructivos para el eucalipto rojo estudiado.

Al final de los ensayos, los resultados fueron analizados por medio de la prueba $t$ con grado de significancia a 5\% ( $P$-valor $<0,05)$, para investigar la influencia del contenido de humedad (30 y 12\%) en las propiedades físico-mecánicas estudiadas. Por la formulación de hipótesis, el P-valor mayor que el nivel de significación implica aceptar la hipótesis nula (donde las medias de los dos grupos son equivalentes) y rechazarla de otro modo (medias no equivalentes).

\section{Resultados}

Los resultados obtenidos se dividieron en cuatro cuadros de acuerdo con sus propiedades, los cuales incluyen: humedad, repeti- ciones, medias de las propiedades, desviación estándar y el P-valor. En principio, dos propiedades físicas fueron estimadas, densidad aparente y básica, cuyos resultados medios y estadísticos obtenidos están indicados en el Cuadro 2.

Mediante la condición evaluada de reducción del contenido de humedad de 30\% (saturado verde) para el estándar de la norma de $12 \%$ (seco), la densidad aparente disminuyó $16,67 \%\left(0,18 \mathrm{~g} / \mathrm{cm}^{3}\right)$, mientras que la densidad absoluta permaneció totalmente estable, esto es, con el mismo valor de la condición inicial verde (Cuadro 2).

Con respecto a las catorce propiedades mecánicas, los resultados medios y estadísticos de las mismas están indicados en los Cuadros 3,4 y 5 .

Con la reducción de la humedad del estado verde estable (30\%) para el estado estándar estable (12\%) para el empleo en estructuras de madera, todos los módulos de ruptura del eucalipto rojo demostraron aumento en sus propiedades del orden de: 19,17\% (9,2 $\mathrm{MPa})$ para la compresión paralela, 38,71\% $(2,4 \mathrm{MPa})$ en la compresión normal, 26,09\% (1,2 $\mathrm{MPa})$ en la tensión normal, $11,00 \%(14,08 \mathrm{MPa})$ en la tensión paralela, y 39,27\% $(48,1 \mathrm{MPa})$ en la flexión (Cuadro 3).

Por su lado, todavía sobre el estudio de la reducción del contenido de humedad de la madera de Eucalyptus camaldulensis, todos los módulos de elasticidad estudiados también demostraron aumentos en sus propie-

\begin{tabular}{lccccc}
\hline \multicolumn{1}{c}{ Característica } & CH (\%) & n & M & de & P-valor \\
\hline \multirow{2}{*}{ Densidad Aparente $\left(\mathrm{g} / \mathrm{cm}^{3}\right)$} & 30 & 17 & 1,08 & 0,11 & $0,0000^{*}$ \\
\hline \multirow{2}{*}{ Densidad Básica $\left(\mathrm{g} / \mathrm{cm}^{3}\right)$} & 12 & 18 & 0,90 & 0,09 & - \\
& 30 & 17 & 0,71 & 0,12 & \\
CH: contenido de humedad, n: número de repeticiones, M: medias de las propiedades, de: desviación \\
estándar, *: P $<0,05$ en prueba t
\end{tabular}

Cuadro 2. Resultados para las densidades de la madera de Eucalyptus camaldulensis. 


\begin{tabular}{lccccc}
\hline \multicolumn{1}{c}{ Característica } & CH $(\%)$ & $\mathbf{n}$ & M & de & P-valor \\
\hline \multirow{2}{*}{ Compresión Paralela (MPa) } & 30 & 17 & 38,8 & 5,1 & $0,0000^{*}$ \\
& 12 & 18 & 48,0 & 6,3 & \\
\hline \multirow{2}{*}{ Compresión Normal (MPa) } & 30 & 16 & 3,8 & 1,7 & $0,0048^{*}$ \\
\hline \multirow{2}{*}{ Tensión Paralela (MPa) } & 12 & 18 & 6,2 & 2,8 & \\
\hline \multirow{2}{*}{ Tensión Normal (MPa) } & 30 & 15 & 67,1 & 32,5 & \multirow{2}{*}{$0,3088^{*}$} \\
& 12 & 14 & 78,1 & 24,2 & \\
\hline \multirow{2}{*}{ Flexión Estática (MPa) } & 30 & 16 & 3,4 & 1,5 & \multirow{2}{*}{$0,0310^{*}$} \\
& 12 & 18 & 4,6 & 1,6 & \\
\hline CH: con & 30 & 15 & 74,4 & 8,0 & \multirow{2}{*}{$0,0068^{*}$} \\
\hline
\end{tabular}

$\overline{\mathrm{CH}}$ : contenido de humedad, n: número de repeticiones, M: medias de las propiedades, de: desviación estándar, *: $\mathrm{P}<0,05$ en prueba $\mathrm{t}$

Cuadro 3. Resultados para los módulos de ruptura del Eucalyptus camaldulensis.

\begin{tabular}{lccccc}
\hline \multicolumn{1}{c}{ Característica } & CH (\%) & n & M & de & P-valor \\
\hline \multirow{2}{*}{ Compresión Paralela (MPa) } & 30 & 17 & 12396,7 & 3954,8 & \multirow{2}{*}{$0,5254^{*}$} \\
& 12 & 18 & 13286,1 & 4242,8 & \\
\hline \multirow{2}{*}{ Compresión Normal (MPa) } & 30 & 16 & 380,4 & 173,9 & \multirow{2}{*}{$0,0061^{*}$} \\
\hline \multirow{2}{*}{ Tensión Paralela (MPa) } & 12 & 18 & 616,1 & 283,3 & \\
\hline \multirow{2}{*}{ Flexión Estática (MPa) } & 30 & 15 & 13532,2 & 5635,9 & \multirow{2}{*}{$0,0625^{*}$} \\
& 12 & 14 & 17351,0 & 4939,3 & \\
\hline
\end{tabular}

CH: contenido de humedad, n: número de repeticiones, M: medias de las propiedades, de: desviación estándar, *: $\mathrm{P}<0,05$ en prueba $\mathrm{t}$

Cuadro 4. Resultados para los módulos de elasticidad del Eucalyptus camaldulensis.

dades, por ejemplo, en la compresión paralela $(6,69 \%$ o $889,4 \mathrm{MPa})$, en la compresión normal $(38,26 \%$ o $235,7 \mathrm{MPa})$, en la tensión paralela $(22,01 \%$ o $3818,8 \mathrm{MPa})$ y en la flexión estática (16,95\% o $2523,3 \mathrm{MPa}$ ) (Cuadro 4).

De modo similar a los resultados obtenidos de los módulos de ruptura y elasticidad, las cinco últimas propiedades de resistencia de la madera de Eucalyptus camaldulensis también indicaron aumentos en sus propiedades como: $25,99 \%(4,6 \mathrm{MPa})$ en el cizallamiento, $36,21 \%$ $(0,42 \mathrm{MPa})$ en el agrietamiento, $22,60 \%(1,91$ $\mathrm{kN})$ en la dureza normal, $22,52 \%(1,79 \mathrm{kN})$ en la dureza paralela y $23,48 \%(2,7$ N.m) en la tenacidad (Cuadro 5).

\section{Discusión}

Acerca del análisis de la prueba t a los valores obtenidos para la madera de Eucalyptus camaldulensis, se verifica que la propiedad de la densidad aparente sufre variación con la reducción del contenido de humedad de $30 \%$ para $12 \%$ $(P$-valor $<0,05)$. Sin embargo, esta situación no ocurre con la densidad básica del Eucalyptus camaldulensis, ya que esta propiedad no sufre variación con la reducción de humedad (Cuadro 2). Los valores de las densidades para la madera seca de eucalipto rojo son similares a la bibliografía, por ejemplo, la densidad básica de $0,683 \mathrm{~g} / \mathrm{cm}^{3}$ (Sturion et al. 1987) y la densidad aparente de $0.98 \mathrm{~g} / \mathrm{cm}^{3}$ (FAO/UN 1981). 


\begin{tabular}{lccccc}
\hline Característica & CH $(\%)$ & n & M & de & P-valor \\
\hline \multirow{2}{*}{ Cizallamiento (MPa) } & 30 & 17 & 12,1 & 1,6 & $0,0000^{*}$ \\
\hline \multirow{2}{*}{ Agrietamiento (MPa) } & 12 & 18 & 15,3 & 2,0 & \\
\hline \multirow{2}{*}{ Dureza Normal (kN) } & 30 & 15 & 0,74 & 0,19 & \multirow{2}{*}{$0,0000^{*}$} \\
\hline \multirow{2}{*}{ Dureza Paralela (kN) } & 12 & 18 & 1,16 & 0,24 & \\
\hline \multirow{2}{*}{ Tenacidad (N.m) } & 30 & 17 & 6,54 & 0,89 & \multirow{2}{*}{$0,0000^{*}$} \\
& 12 & 18 & 8,45 & 1,22 & \\
\hline CH: cons & 30 & 17 & 6,16 & 1,27 & \multirow{2}{*}{$0,0002 *$} \\
\hline
\end{tabular}

$\overline{\mathrm{CH}}$ : contenido de humedad, n: número de repeticiones, M: medias de las propiedades, de: desviación estándar, *: $\mathrm{P}<0,05$ en prueba $\mathrm{t}$

Cuadro 5. Resultados para otras propiedades de resistencia del Eucalyptus camaldulensis.

En relación con los módulos de ruptura, mediante la prueba $t$, se verificó que las propiedades de compresión paralela y normal, tensión normal y flexión estática sufrieron influencias en sus propiedades cuando estas fueron sometidas a la reducción precipitada del contenido de humedad $(P$-valor $<0,05)$. Esta situación no fue observada en la propiedad de tensión paralela (Cuadro 3), posiblemente debido a los factores anatómicos de la madera de eucalipto rojo, como por ejemplo, las vetas entrecruzadas y bolsas de resina presentes en esta especie, tal como apuntan Wallis (1970), FAO/UN (1981) y Boland et al. (2006). Siendo así, el análisis estadístico no corroboró el incremento mecánico esperado con la reducción de la humedad.

Para los módulos de elasticidad, en el mismo análisis de la prueba $t$, aún se verifica que la compresión normal y la flexión estática tienen influencias en sus propiedades cuando son sometidas a la reducción de humedad de $30 \%$ para $12 \%(P$-valor $<0,05)$. La compresión paralela y la tensión paralela no sufren influencias en sus propiedades, esto es, no ocurre el rechazo de la hipótesis nula de la igualdad de las medias en esas condiciones citadas (Cuadro 4).

Para los valores obtenidos para la madera de eucalipto rojo, se verifica en la prueba t que las propiedades de dureza normal y paralela, cizallamiento y agrietamiento sufren influen- cia en los valores de sus propiedades cuando son sometidas a la reducción del contenido de humedad para $12 \%$ ( $P$-valor $<0,05)$. No obstante, la tenacidad no sufre influencia en sus valores cuando la misma es sometida a la reducción de humedad de las muestras probadas (Cuadro 5).

Así, todas las diez propiedades que sufrieron influencia en sus valores demostraron aumentos en resistencia. Esa mejora en la mayoría de las propiedades mecánicas evaluadas de la madera de Eucalyptus camaldulensis, en condición seca, contribuye para la utilización eficiente para fines estructurales. Además, según la norma brasileña NBR 7190 (1997), esos resultados colocan la madera de Eucalyptus camaldulensis de plantaciones como una especie forestal eficiente para las estructuras de madera, por respetar los valores de resistencia prescriptos para construcción.

En comparación con las especies nativas de densidad básica similar, es decir, alrededor de $700 \mathrm{~kg} / \mathrm{m}^{3}$, fue posible indicar que los resultados obtenidos para eucalipto rojo en la condición verde fueron menores que los informados por Nahuz (2013) para las especies brasileñas Cupiúba (Goupia glabra Aubl.), Curipixá (Micropholis venulosa (Mart. \& Eichler) Pierre), Garapa (Apuleia leiocarpa (J. Vogel) J. F. Macbr.) y Tatajuba (Bagassa guianensis Aubl.), por 
cuenta de, según Boland et al. (2006), la presencia de bolsas de resina que reducen su resistencia a la ruptura. Entonces, por medio de los resultados obtenidos, el uso estructural de la madera de Eucalyptus camaldulensis es interesante, pues, de acuerdo con Foroughbakhch et al. (2017), ese tipo de madera "puede contribuir para el aumento de la madera explotada en la industria y para la diminución de la presión sobre las especies nativas", visto que tales especies naturales aún son, según De Araujo et al. (2016), ampliamente utilizadas en la construcción civil de habitaciones en todo el mundo, incluyendo muchos países europeos que adquieren kits prefabricados de viviendas en madera nativa brasileña.

En comparación con el estudio igualmente enfocado para aplicación estructural, de Nogueira et al. (2018) para la madera de Eucalyptus umbra R. T. Baker, la misma tuvo un resultado bastante similar a la madera de Eucalyptus camaldulensis aquí estudiada y de densidad muy equivalente, donde trece propiedades mecánicas fueron ligeramente mejores para el contenido de humedad de $30 \%$ y sólo cinco de esas propiedades presentaron resultados un poco mejores en la condición de $12 \%$. Así, la madera de Eucalyptus camaldulensis (Cuadros 3 a 5) indicó un rendimiento mecánico sensiblemente mejor que el Eucalyptus umbra para el contenido de humedad de $12 \%$, lo cual es más adecuado para las estructuras de madera, según la norma brasileña NBR 7190 (1997).

Por su parte, para una madera de menor densidad, Eucalyptus urophylla S. T. Blake, la cual fue estudiada por Lahr et al. (2017) con objetivo similar para las estructuras, el Eucalyptus camaldulensis tuvo un rendimiento mecánico mucho mayor, visto que presentó menores valores de resistencia sólo en tres y cuatro propiedades para las condiciones de 30 y $12 \%$ de humedad, respectivamente.

\section{Conclusiones}

Entre las propiedades físicas evaluadas de la madera de Eucalyptus camaldulensis procedente de plantación, se averigua que, con la reducción del contenido de humedad de $30 \%$ para $12 \%$ estándar de la norma consultada, la densidad básica se mantiene totalmente estable como se esperaba y la densidad aparente indica un decrecimiento, por cuenta de la pérdida de agua.

En relación a las catorce propiedades mecánicas de la madera de Eucalyptus camaldulensis, diez propiedades indican mejoras en sus propiedades cuando los mismos son sometidos a la reducción citada del contenido de humedad. Así, apenas la tensión paralela en el módulo de ruptura, las compresiones y tensión paralelas en los módulos de elasticidad y la tenacidad no tuvieron cambios en sus resistencias. Las catorce propiedades mecánicas presentaron valores conformes a la norma brasileña, factor que refuerza el uso de esa especie en la construcción.

En virtud del incremento de la mayor parte de sus propiedades de resistencia, con la reducción de la humedad analizada, la cual es reglamentada para el uso confiable y seguro de la madera de Eucalyptus camaldulensis en la construcción civil, se verifica que esta especie tiene grandes posibilidades de uso en toda la América Latina, especialmente en pisos, muebles, también como en componentes y elementos estructurales para construcciones ligeras y pesadas.

\section{Bibliografía}

Abd-Alla, MF; El-Negoumy, SI; El-Lakany, H; Saleh, NAM. 1980. Flavonoid glycosides and the chemosystematics of Eucalyptus camaldulensis. Phytochemistry 19(2):2629-2632.

ABNT (Associação Brasileira de Normas Técnicas). 1997. NBR 7190 - Projeto de estruturas de madeira. Rio de Janeiro, Brasil, ABNT.

Acuña, CV; Villalba, P; Hopp, E; Poltri, SNM. 2014. Transferability of microsatellite markers located in candidate genes for wood properties between Eucalyptus species. Forest Systems 23(3):506-512.

Ashraf, A; Sarfraz RA; Mahmood, A; ud Din, M. 2015. Chemical composition and in vitro 
antioxidant and antitumor activities of Eucalyptus camaldulensis Dehn. leaves. Industrial Crops and Products 74:241-248.

Aytar, F. Dağdaş, S; Duran, C. 2011. Australian insects affecting Eucalyptus species in Turkey. Silva Lusitana 19:41-47.

Bedi, NG; Nawange, SR; Singh, SM; Naidu, J; Kavishwar, A. 2012. Seasonal prevalence of Cryptococcus neoformans var. grubii and Cryptococcus gattii inhabiting Eucalyptus tereticornis and Eucalyptus camaldulensis trees in Jabalpur City of Madhya Pradesh, Central India. Journal de Mycologie Médicale 22(4):341-347.

Bell, DT; van der Moezel, PG; Bennett, IJ; McComb, JA; Wilkins, CA; Marshall, SCB; Morgan, AL. 1993. Comparisons of growth of $\mathrm{Eu}$ calyptus camaldulensis from seeds and tissue culture: root, shoot and leaf morphology of 9-month-old plants grown in deep sand and sand over clay. Forest Ecology and Management 57(1-4):125-139.

Boland, DJ; Brooker, MIH; Chippendale, GM; Hall, N; Hyland, BPM; Johnston, RD; Kleinig, DA; McDonald, MW; Turner, JD. 2006. Forest trees of Australia. 5. ed. Collingwood, Austrália, Csiro Publishing.

Bush, D; Marcar, N; Arnold, R; Crawford, D. 2013. Assessing genetic variation within Eucalyptus camaldulensis for survival and growth on two spatially variable saline sites in southern Australia. Forest Ecology and Management 306:68-78.

Catelotti, K; Kingsford, RT; Bino, G; Bacon, P. 2015. Inundation requirements for persistence and recovery of river red gums (Eucalyptus camaldulensis) in semi-arid Australia. Biological Conservation 184:346-356.

Chingaipe, TM. 1985. Early growth of Eucalyptus camaldulensis under agroforestry conditions at Mafiga, Morogoro, Tanzania. Forest Ecology and Management 11(4):241-244.

Conde, E; Cadahia, E; García Vallejo, MC; DIEZ, R. 1996. Composición polifenólica de madera, corteza y hojas de Eucalyptus camaldulensis, E. globulus y E. rudis. Investi- gación agraria. Sistemas y Recursos Forestales 5(2):333-350.

Costa, ACS; Leal, CS; Santos, LC; Carvalho, AMML; Oliveira, AC; Pereira, BLC. 2017. Propriedades da madeira de cerne e alburno de Eucalyptus camaldulensis. Ciência da Madeira 8(1):10-20.

De Araujo, VA; Vasconcelos, JS; Cortez-Barbosa, J; Morales, EAM; Gava, M; Savi, AF; Garcia, JN. 2016. Wooden residential buildings - a sustainable approach. Bulletin of the Transilvania University of Braşov Series II: Forestry • Wood Industry - Agricultural Food Engineering 9-58(2):53-61.

Dunlop, PJ; Bignell, CM; Hibbert, DB. 2000. Use of gas chromatograms of essential leaf oils to compare clones of Eucalyptus camaldulensis. Biochemical Systematics and Ecology 28(4):383-391.

Durand, N; Rodrigues, JC; Mateus, E; Boavida, C; Branco, M. 2011. Susceptibility variation in Eucalyptus spp. in relation to Leptocybe invasa and Ophelimus maskelli (Hymenoptera: Eulophidae), two invasive gall wasps occurring in Portugal. Silva Lusitana 19:19-31.

Dzikiti, S; Gush, MB; Le Maitre, DC; Maherry, A; Jovanovic, NZ; Ramoelo, A; Cho, MA. 2016. Quantifying potential water savings from clearing invasive alien Eucalyptus camaldulensis using in situ and high resolution remote sensing data in the Berg River Catchment, Western Cape, South Africa. Forest Ecology and Management 361:69-80.

FAO/UN (Food and Agriculture Organization of United Nations). 2011. Agrovoc: Eucalyptus camaldulensis. Consultado 19 mar. 2018. Disponible en: http://artemide.art.uniroma2. it:8081/agrovoc/agrovoc/en/page/c 2685?clang=es.

FAO/UN (Food and Agriculture Organization of United Nations) 1981. Eucalypts for planting. 2. ed. Roma, Itália. FAO/UN.

Ferreira, M. 1979. Escolha de espécies de eucalipto. Circular Técnica IPEF (47):1-30.

Foroughbakhch, R; Carrillo-Parra, A; Hernández-Piñero, JL; Guzmán-Lucio, MA. 2017. 
Growth and yield of an eucalyptus subtropical plantation in a Northeastern Mexico degraded land soil. Madera y Bosques 23(3):71-85.

García Meza, HA; Chumbimune Vivanco, SY; Acevedo Mallque, MP; Chavesta Custodio, M; Cuellar Bautista, JE; Salazar Hinostroza, EJ. 2017. Aptitud de uso del ulcumano (Retrophyllum rospigliosii (Pilg.) C.N. Page), procedente de una plantación de 32 años, mediante la caracterización tecnológica y anatómica. Revista Forestal del Perú 32(2):78-88.

Giamakis, A; Kretsi, O; Chinou, I; Spyropoulos, CG. 2001. Eucalyptus camaldulensis: volatiles from immature flowers and high production of 1,8 -cineole and $\beta$-pinene by in vitro cultures. Phytochemistry 58(2):351-355.

Gonçalves, MR; Passos, CAM. 2000. Crescimento de cinco espécies de eucalipto submetidas a déficit hídrico em dois níveis de fósforo. Ciência Florestal 10(2):145-161.

González, DP; Moglia, JG; López, AJ; Pece, M; López, JA; Moreno, R. 2014. Estimación de las tensiones de crecimiento en individuos selectos de Eucalyptus camaldulensis mediante extensómetro e índice de rajado. Quebracho 22(1,2):57-65.

Heidari, A; Younesi, H; Rashidi, A; Ghoreyshi, A. 2014. Adsorptive removal of CO2 on highly microporous activated carbons prepared from Eucalyptus camaldulensis wood: effect of chemical activation. Journal of the Taiwan Institute of Chemical Engineers 45(2):579-588.

Hunter, I. 2001. Above ground biomass and nutrient uptake of three tree species (Eucalyptus camaldulensis, Eucalyptus grandis and Dalbergia sissoo) as affected by irrigation and fertiliser, at 3 years of age, in southern India. Forest Ecology and Management 144(1-3):189-199.

IICA (Inter-American Institute for Cooperation on Agriculture). 2007. USDA National Agricultural Library: Eucalyptus camaldulensis. Consultado 19 mar. 2018. Disponible en https://agclass.nal.usda.gov/mtwdk.exe?k=default $\& \mathrm{l}=60 \& \mathrm{n}=1 \& \mathrm{~s}=5 \& \mathrm{t}=2 \& \mathrm{w}=38357$.

Ikka, T.; Ogawa, T; Li, D; Hiradate, S; Morita, A. 2013. Effect of aluminum on metabolism of organic acids and chemical forms of aluminum in root tips of Eucalyptus camaldulensis Dehnh. Phytochemistry 94:142-147.

Kabay, ED; Thomson, LAJ; Doran, JC; van der Moezel, PG; Bell, DT; McComb, JA; Bennett, LJ; Hartney, VJ; Malajczuk, N. 1986. Micropropagation of forest trees selected for salt tolerance. Australian Salinity Newsletter 14:6062.

Knezevic, P; Aleksic, V; Simin, N; Svircev, E; Petrovic, A; Mimica-Dukic, N. 2016. Antimicrobial activity of Eucalyptus camaldulensis essential oils and their interactions with conventional antimicrobial agents against multi-drug resistant Acinetobacter baumannii. Journal of Ethnopharmacology 178:125-136.

Lahr, FAR; Nogueira, MCJA; De Araujo, VA; Vasconcelos, JS; Christoforo, AL. 2017. Physical-mechanical characterization of Eucalyptus urophylla wood. Engenharia Agrícola 37(5):900-906.

Lanfranco, JW; Marlats, RM; Baridon, E. 1999. Vertedero de residuos sólidos urbanos: pedogénesis comparada entre sitios de una plantación de Eucalyptus camaldulensis Dehnh. y de vegetación herbácea naturalizada. Investigación Agraria. Sistemas y Recursos Forestales 8(2):293-304.

Li, S; Chen, F; Jia, J; Liu, Z; Gu, H; Yang, L; Wang, F; Yang, F. 2016. Ionic liquid-mediated microwave-assisted simultaneous extraction and distillation of gallic acid, ellagic acid and essential oil from the leaves of Eucalyptus camaldulensis. Separation and Purification Technology 168:8-18.

Marlats, RM; Pérez, F. 1991. Selección de especies, orígenes y procedencias de Eucalyptus, resistentes a bajas temperaturas en plantas de vivero para el norte de la depresión del salado, provincia de Buenos Aires, Argentina. Investigación Agraria. Sistemas y Recursos Forestales $0: 151-161$.

Medhi, SM; Reza, SA; Mahnaz, K; Reza, AM; Abbas, H; Fatemeh, M; Hassan, V. 2010. Phytochemistry and larvicidal activity of Eucalyptus camaldulensis against malaria vector, Anophe- 
les stephensi. Asian Pacific Journal of Tropical Medicine 3:841-845.

NAHB (National Association of Home Builders). 1996. Tendencias en las construcciones residenciales para mediados de la década de los 90's. Madera y Bosques 2:75-82.

Nahuz, AR. (Coord.) 2013. Catálogo de madeiras brasileiras para a construção civil. São Paulo, Brasil. Instituto de Pesquisas Tecnológicas (IPT). 103 p. Consultado 19 mar. 2018. Disponible en http://www.ipt.br/download. php?filename $=980$-Catalogo de Madeiras Brasileiras para a Construcao Civil.PDF.

Nogueira, MCJA; Almeida, DH; Vasconcelos, JS; Almeida, TH; Araujo VA; Christoforo, AL; Lahr, FAR. 2018. Properties of Eucalyptus umbra wood for timber structures. International Journal of Materials Engineering 8(1):12-15.

Ohshima, J; Yokota, S; Yoshizawa, N; Ona, T. 2005. Representative heights for assessing whole-tree values and the within-tree variations of derived wood properties in Eucalyptus camaldulensis and E. globulus. Wood and Fiber Science 37:51-65.

Okino, EYA; Souza, MR; Santana, MAE; Alves, MVS; Sousa, ME; Teixeira, DE. 2004. Cement-bonded wood particleboard with a mixture of eucalypt and rubberwood. Cement \& Concrete Composites 26:729-734.

Onyewotu, LOZ; Ogigirigi, MA; Stigter, CJ. 1994. A study of competitive effects between a Eucalyptus camaldulensis shelterbelt and an adjacent millet (Pennisetum typhoides) crop. Agriculture, Ecosystems \& Environment 51:281-286.

Ounban, W; Puangchit, L.; Diloksumpun, S. 2016. Development of general biomass allometric equations for Tectona grandis Linn.f. and Eucalyptus camaldulensis Dehnh. plantations in Thailand. Agriculture and Natural Resources 50:48-53.

Pagano, MC; Scotti, MR. 2008. Arbuscular and ectomycorrhizal colonization of two Eucalyptus species in semiarid Brazil. Mycoscience 48:379-384.
Patnukao, P; Pavasant, P. 2008. Activated carbon from Eucalyptus camaldulensis Dehn bark using phosphoric acid activation. Bioresource Technology 99:8540-8543.

Patnukao, P; Kongsuwan, A; Pavasant, P. 2008. Batch studies of adsorption of copper and lead on activated carbon from Eucalyptus camaldulensis Dehn. bark. Journal of Environmental Sciences 20:1028-1034.

Pinyopusarerk, K; Doran, JC; Williams, ER; Wasuwanich, P. 1996. Variation in growth of Eucalyptus camaldulensis provenances in Thailand. Forest Ecology and Management 87:6373.

Pirralho, M; Flores, D; Sousa, VB; Quilhó, T; Knapic, S; Pereira, H. 2014. Evaluation on paper making potential of nine Eucalyptus species based on wood anatomical features. Industrial Crops and Products 54:327-334.

Sabas, E; Nshumbemuki, L. 1988. Eucalyptus camaldulensis provenances for afforestation in Mwanza and Shinyamga regions of Tanzania. Forest Ecology and Management 24:127-138.

Sandoval, S; Cancino, J; Esquivel, E; Acuña, E; Rubilar, R; Espinosa, M. 2015. Evaluation of damage caused by Ectinogonia buquetti (Coleoptera: Buprestidae) in dendroenergetic plantations of Eucalyptus camaldulensis. Bosque 36(2):247-254.

Siddiqui, BS; Sultana, I; Begum, S. 2000. Triterpenoidal constituents from Eucalyptus camaldulensis var. obtusa leaves. Phytochemistry 54:861-865.

Sturion, JA; Pereira, JCD; Albino, JC; Morita, M. 1987. Variação na densidade básica da madeira de doze espécies de Eucalyptus plantadas em Uberaba, MG. Boletim de Pesquisa Florestal (14):28-38.

Sun, D; Dickinson, GR. 1995. Salinity effects on tree growth, root distribution and transpiration of Casuarina cunninghamiana and Eucalyptus camaldulensis planted on a saline site in tropical north Australia. Forest Ecology and Management 77:127-138.

Teixeira, PC; Leal, PGL; Barros, NF; Novais, RF. 1995. Nutrición potásica y relaciones 
hídricas en plantas de Eucalyptus spp. Bosque 16(1):61-68.

Tyynelä, TM. 2001. Species diversity in Eucalyptus camaldulensis woodlots and miombo woodland in Northeastern Zimbabwe. New Forests 22:239-257.

Verdeguer, M; Amparo Blázquez, M; Boira, $\mathrm{H}$. 2009. Phytotoxic effects of Lantana camara, Eucalyptus camaldulensis and Eriocephalus africanus essential oils in weeds of Mediterranean summer crops. Biochemical Systematics and Ecology 37:362-369.

Wallis, NK. 1970. Australian timber handbook. 3. ed. Sydney, Austrália, Angus \& Robertson.

Presentado: 19/03/2018

Aceptado: 22/06/2018 